DGfK-Nachrichten

\section{DGfK-Präsident: \\ Aus der Arbeit des Vorstandes}

Liebe DGfK-Mitglieder, liebe Leserinnen und Leser,

gerade die Urlaubszeit bietet der Kartographie hervorragende Gelegenheit, weiten Teilen der Bevölkerung die besonderen Qualitäten kartographischer Gestaltung im wahrsten Sinne des Wortes vor Augen zu führen. Zu keiner Zeit des Jahres nutzen so viele Menschen Karten aller Art, kartenverwandte Darstellungen in ihrer ganzen Vielfalt, kartenbasierte Informationssysteme, Navigationssysteme und Routenplaner für private Zwecke. Für viele beginnt die Urlaubsplanung bereits mit Kartographie - zur Suche des Urlaubsortes, zur Erkundung seiner Umgebung, zur Vorbereitung der Reise. Am Urlaubsort selbst werden der touristische Stadtplan vom Abreiß-Bock am Hoteltresen, die Tafel mit Wanderwegen am Waldparkplatz oder die reiß- und wasserfeste Radwanderkarte zur idealen Informationsquelle bei der Suche nach bekannten Sehenswürdigkeiten, malerischen Landschaften oder idyllischen Gasthöfen.

Doch nicht immer gelingt die Informationsgewinnung wie erhofft. Wir alle kennen die Bilder von Touristen in Großstädten, mit zerknitterten Stadtplänen ihr Ziel suchend, von Radfahrern an Kreuzungen, bemüht den Inhalt ihrer Karte mit der Beschilderung am Straßenrand in Übereinstimmung zu bringen, oder auch von Nutzern mobiler Endgeräte auf der Suche nach Schatten, um auf dem kontrastarmen Display irgendetwas erkennen zu können. Oft lassen sich Probleme bei der Nutzung räumlicher Darstellungen durch kartographischen Sachverstand bei der Herstellung vermeiden. Manchmal genügt bereits der Rückgriff auf

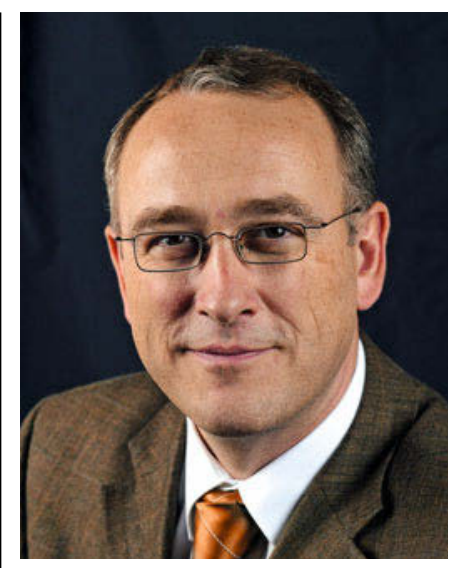

Prof. Dr. Manfred Weisensee

einfaches Lehrbuchwissen, um aus einem unübersichtlichen Liniengewirr eine leicht verständliche Karte zu generieren.

Innerhalb der DGfK wurde in den vergangenen Monaten - gerade zuletzt im Rahmen des DKT in Hamburg - in verschiedenen Diskussionen dieser kartographische Sachverstand als ein Alleinstellungsmerkmal unserer Mitglieder genannt. Weiterhin wurde thematisiert, dass ebendiese Kompetenz auch deutlich kommuniziert werden müsse - sowohl innerhalb der Geo-Fachverbände als auch in andere Bereiche, welche sich mit der Darstellung raumbezogener Informationen beschäftigen.

Wir werden in nächster Zeit reichlich Gelegenheit haben, Konsequenzen aus diesen Diskussionen zu ziehen und gerade der Fachöffentlichkeit die besonderen Qualitäten kartographischer Gestaltung deutlich zu machen. Im Rahmen des Verbändeparks der Intergeo des DVW wird die DGfK in diesem Jahr in Berlin wieder präsent sein und die Ergebnisse der Arbeit ihrer Kommissionen sowie eine Neuauflage der Image-Broschüre der DGfK vorstellen. Mit dem DDGI werden wir dort auch die Möglichkeiten diskutieren, dessen neue InternetPlattform Allianz Geoinformation als weiteres Informations- und Kommunikationsforum für unsere Mitglieder zu nutzen. Und für den Januar 2015 ist eine Neuauflage der Tagung News - Infographics - Maps in der Sektion Berlin-Brandenburg geplant, um den
Austausch zwischen Kartographie und Infografik zu intensivieren.

Die Planungen für den Deutschen Kartographentag im Jahr 2016 wurden mit der Sektion Berlin-Brandenburg und der Geomatik Tagungs $\mathrm{GmbH}$ im Rahmen einer Vorstandssitzung in Berlin ebenfalls vorangetrieben. Dort wurde beschlossen, den DKT 2016 vom 14. bis 16. Juni im Kongresshotel Potsdam am Templiner See durchzuführen. Das Kongresshotel bietet ideale Voraussetzungen für eine erfolgversprechende Fachtagung und ebenso die Räumlichkeiten für eine ansprechende Fachausstellung. Die Lage des Hotels in der Landeshauptstadt Potsdam und die direkte Verbindung in die Bundeshauptstadt Berlin bieten vielfältige Möglichkeiten gleichermaßen für Fachexkursionen und Freizeitgestaltung.

Im Rahmen der Vorstandssitzung in Berlin wurde Manfred Buchroithner in Anerkennung seiner Verdienste um die Ausrichtung der 26. Internationalen Kartographischen Konferenz From Pole to Pole 2013 in Dresden mit einem Ehrenbrief ausgezeichnet. Mit dem Ehrenbrief würdigt die DGfK vorbildliche Einzelleistungen von Mitgliedern in besonderen Projekten. Es ist das große Verdienst Manfred Buchroithners, dass die 26. Internationale Kartographische Konferenz der Internationalen Kartographischen Vereinigung überhaupt in Dresden und dann mit so großem Erfolg durchgeführt werden konnte. Manfred Buchroithner hat als Kongresspräsident beharrlich an der Realisierung dieses Ereignisses gearbeitet. Und auch der 61. Deutsche Kartographentag in Dresden wurde durch die Anbindung an diesen internationalen Kongress zu einem ganz besonderen Ereignis.

Ich wünsche Ihnen im Namen der Deutschen Gesellschaft für Kartographie e.V. (DGfK) - Gesellschaft für Kartographie und Geomatik eine erholsame Urlaubszeit.

Manfred Weisensee, Hude Präsident der DGfK
Iran-Exkursion der Sektion Halle/Leipzig der DGfK

Vom 1. bis 17. März 2014 reisten Mitglieder der Sektion und Teilnehmer aus den Sektionen Dresden, Mittelrhein, Rhein Ruhr und Thüringen zusammen mit Gästen unter Leitung von M. Linke nach Iran. Es war die 5. Exkursion dieser Art, und fiir eine finanzielle Unterstützung bei der Anfertigung des Exkursionsfuhrers danken wir wieder unserer Sektionsleitung. Die Reise begann in Tehran und führte zunächst zum Orumiyehsee (früher Urmia), dessen Wasserfläche durch zu starke Nutzung seiner östlichen Zuflüsse für landwirtschaftliche Zwecke stark zurückgegangen ist. Von dort führte die Fahrt zum historischen Observatorium nach Maragheh, von dem sich ein Globus seit 1562 im Mathematisch-Physikalischen Salon in Dresden befindet, zum Kaspisee, und von dort flogen wir nach Ahvaz in das Arvanddelta (Shatt el-Arab), von hier aus besuchten wir Susa und die großartigen Relikte aus elamischer Zeit. Dabei erschloss sich uns ein hohes touristisches Potential des Landes, das bei einer guten Infrastruktur mit ausgezeichneten Hotels und Straßen mit viel Autobahnen leider viel zu wenig von Ausländern genutzt wird. Weiter ging es über Busher nach Schiraz mit den höchst sehenswerten Ruinen von Persepolis und dem Hafisgrab in der Stadt selbst, was Anlass bot, am Grab aus Goethes West-östlichen Diwan und aus Hafis Schriften zu zitieren. Weiter ging es in die berühmte und früher gefiirchtete Wüste Lut, die heute auf modernen Straßen gefahrlos durchquert werden kann und von da aus nach Yazd und Isfahan, wohl eine der schönsten Städte des Landes, "Isfahan ist die halbe Welt" sagen die Iraner. Den Abschluß der Reise bildete ein höchst anregendes Gespräch mir iranischen Geistlichen in Qom über die Rolle der Frau im Iran und weitere interessante Fragen. Auffällig war die große Freundlichkeit und 